\title{
Hallucinations : images mentales et imagerie cérébrale fonctionnelle
}

\section{Viewing hallucinations with cerebral functional imaging}

\section{Claire RASCLE*, Pierre THOMAS, Michel GOUDEMAND}

Clinique Fontan, CHRU de Lille - 59037 LILLE CEDEX

*Auteur à qui adresser la correspondance : Claire RASCLE, Clinique Fontan, Service de Psychiatrie Générale, CHRU de Lille, 6, rue du Pr Laguesse - 59037 LILLE CEDEX Tél : 0320444584 - Fax : 0320446265 - E-mail : crascle@chru-lille.fr

\section{RÉSUMÉ}

L'hallucination, définie comme une perception sans objet à percevoir, entre dans la littérature psychiatrique au XIXe siècle. Outre les causes psychiatriques, un certain nombre de pathologies neurologiques, de causes toxiques, de situation de privation sensorielle, d'états de conscience modifiée peuvent être à l'origine de phénomènes hallucinatoires. Comme le suggère le mode d'action des substances hallucinogènes, d'une part, et des neuroleptiques anti-hallucinatoires, d'autre part, les systèmes dopaminergique et sérotoninergique sont impliqués dans la genèse des hallucinations, mais leur rôle précis n'est pas encore clairement établi. Les techniques d'imagerie fonctionnelle offrent lès moyens de visualiser le fonctionnement cérébral au moment de la production de ces phénomènes riches et complexes, et d'étudier, en s'appuyant sur les modèles de l'action issus de la psychologie cognitive, les hallucinations décrites par les patients atteints de psychose. Les hallucinations ont un support biologique dans le cerveau qui les subit.

\section{MOTS-CLÉS}

Hallucination, schizophrénie, IRMf, cortex auditif, perception du langage.

\section{SUMMARY}

Hallucinations are sensory perceptions in the absence of external stimuli. They have multiple aetiologies (neurological, toxic, psychiatric). Auditory hallucinations (especially voices) are by far the most common type of hallucination in schizophrenia. Dopaminergic and serotoninergic systems are involved in their genesis. Functional imaging techniques offer the opportunity to see all the varieties of these complex phenomenon in the living brain and to study cognitive models of self monitoring underlying hallucinations in schizophrenics.

\section{KEY-WORDS}

Hallucination, schizophrenia, fMRI, auditory cortex, speech perception. 


\section{Introduction}

Une hallucination est une perception ou une sensation survenant alors qu' aucune stimulation externe ou interne n'affecte les terminaisons nerveuses sensorielles. Le sujet souffrant d'un tel phénomène se méprend sur la perception de son environnement ou de son propre corps. Les hallucinations peuvent intéresser les cinq sens ainsi que simuler des informations perceptives en provenance de l'appareil locomoteur ou phonatoire (hallucinations motrices) ou des viscères (hallucinations cénesthésiques). Les pathologies mentales, en particulier la schizophrénie, les lésions cérébrales focalisées comme d'autres maladies neurologiques (migraines, épilepsies), mais aussi la déprivation sensorielle, le manque de sommeil et la consommation de toxiques peuvent être à l'origine de toutes formes d'hallucinations, depuis la sensation la plus élémentaire, jusqu'a l'audition du langage. Après une brève présentation des aspects cliniques des phénomènes hallucinatoires, nous illustrerons, au travers d'études en imagerie cérébrale fonctionnelle, les hypothèses explicatives actuelles de ces phénomènes.

\section{Aspect clinique}

La prévalence des hallucinations en population générale serait comprise entre 10 et $40 \%$ (1). Les symptômes hallucinatoires, si souvent observés dans la schizophrénie, ont été décrits de manière extensive (2). Ils surviendraient chez $60 \%$ des patients atteints de schizophrénie. Sources de handicap important, ils sont à l'origine d'une désinsertion sociale et professionnelle, rapide et durable. Un quart seraient résistants aux traitements pharmacologiques et deviendraient chroniques (3). Les hallucinations les plus fréquentes concernent les modalités auditives et le langage (voix). Dans certains cas, les voix qu'entend un schizophrène commentent simplement ses actes, dans d'autres cas, elles lui donnent des ordres. Leur perception peut être désagréable. Elles peuvent s'imposer au sujet. Le sujet peut également provoquer ou contrôler ses hallucinations. Un même individu peut expérimenter ces différents types d'hallucinations.

\section{Les hypothèses explicatives}

Devant une telle diversité clinique, peut-on trouver des parallèles et/ou des points communs entre tous ces phénomènes ? La plupart des réponses viennent des études en imagerie cérébrale fonctionnelle ${ }^{1}$, effectuées chez le sujet schizophrène et de données pharmacologiques sur les dérivés du LSD. Les hallucinations ont un support biologique dans le cerveau de celui qui les subit (3).

L'observation des patients schizophrènes sous l'emprise d'hallucinations a montré une activation anormale de l'ensemble des régions correspondant à la modalité sensorielle des hallucinations en question. Le cortex visuel s'active dans le cas d'hallucinations visuelles (4), le cortex auditif dans celui d'hallucinations auditives (5). L'étude de Lennox et coll. (4) réalisée chez quatre patients schizophrènes expérimentant des hallucinations auditives pendant la réalisation d'une IRM fonctionnelle (3 Teslas), soutient l'hypothèse d'une activation anormale des voies normales de l'audition. La Figure 1 montre que l'activation du gyrus temporal supérieur droit et gauche, du cortex pariétal inférieur gauche et du gyrus frontal moyen gauche pendant les périodes d'hallucinations auditives, comparées aux périodes sans hallucinations, chez des patients atteints de schizophrénie.

Les mêmes aires auditives primaires sont activées au cours des hallucinations, que lors de l'écoute de stimuli verbaux. L'activation des aires frontales serait soit en lien avec la nature émotionnelle de l'hallucination, soit le rappel de mémoire verbale activant des zones cérébrales identiques.

Cette suractivité du cortex cérébral rendrait ces phénomènes très proches de ceux expérimentés par les utilisateurs de $\mathrm{LSD}^{2}$. Dans ce cas, les hallucinations proviennent d'une interprétation erronée par le cerveau de messages provenant des yeux et des oreilles. Dans le cas de la schizophrénie, ces hallucinations surviennent sans stimulation sensorielle. Elles diffèrent, également, $\mathrm{du}$ fruit de notre imagination qui nous permet en fermant les yeux d'imaginer par exemple la place St Marc ou de dialoguer dans sa tête avec un interlocuteur absent. Dans ces deux circonstances, l'aspect imaginaire est évident. Ce qui n'est pas le cas pour un patient schizophrène qui aura l'impression, dans ce type de situation, d'entendre des voix. Cette perte de contrôle serait expliquée par un trouble de la connectivité cortico-corticale (3). L'hypothèse d'un trouble du contrôle du langage intérieur, qui entraînerait chez le patient une confusion, entre le discours qu'il produit et les paroles entendues, a été étudiée. Un trouble des connexions

\footnotetext{
'Imagerie cérébrale fonctionnelle : on regroupe sous cette terminologie les techniques d'imagerie médicale (potentiels évoqués cognitifs, imagerie par émission de position, débitmétrie cérébrale, résonance magnétique nucléaire d'activation cérébrale, etc...) explorant les aspects fonctionnels de l'activité cérébrale en opposition avec l'imagerie cérébrale morphologique (tomodensitométrie; résonance magnétique nucléaire de structure).

${ }^{2} L S D$; le diéthylamide de l'acide lysergique (un des constituants de l'alcaloïde de l'ergot de seigle), est une drogue psychodysleptique, activant le système sérotoninergique, synthétisée en 1958 par Hofmann.
} 
$\mathbf{a}$

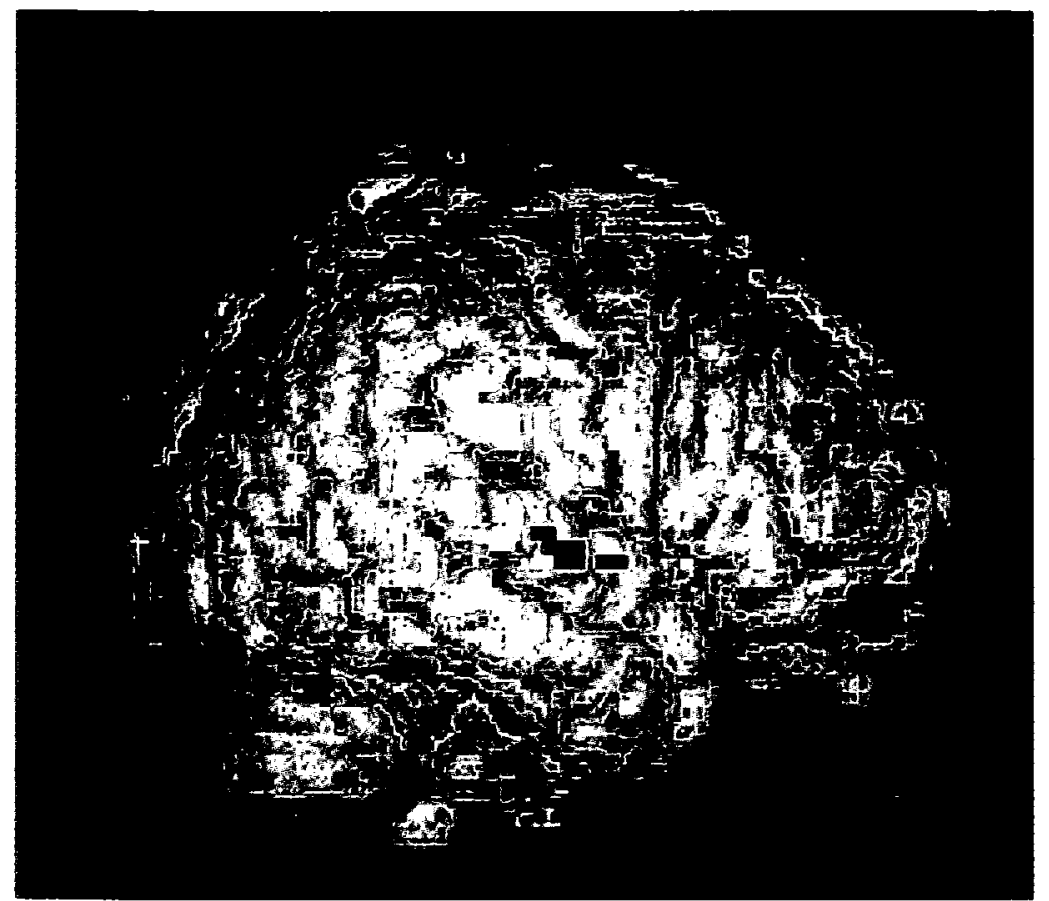

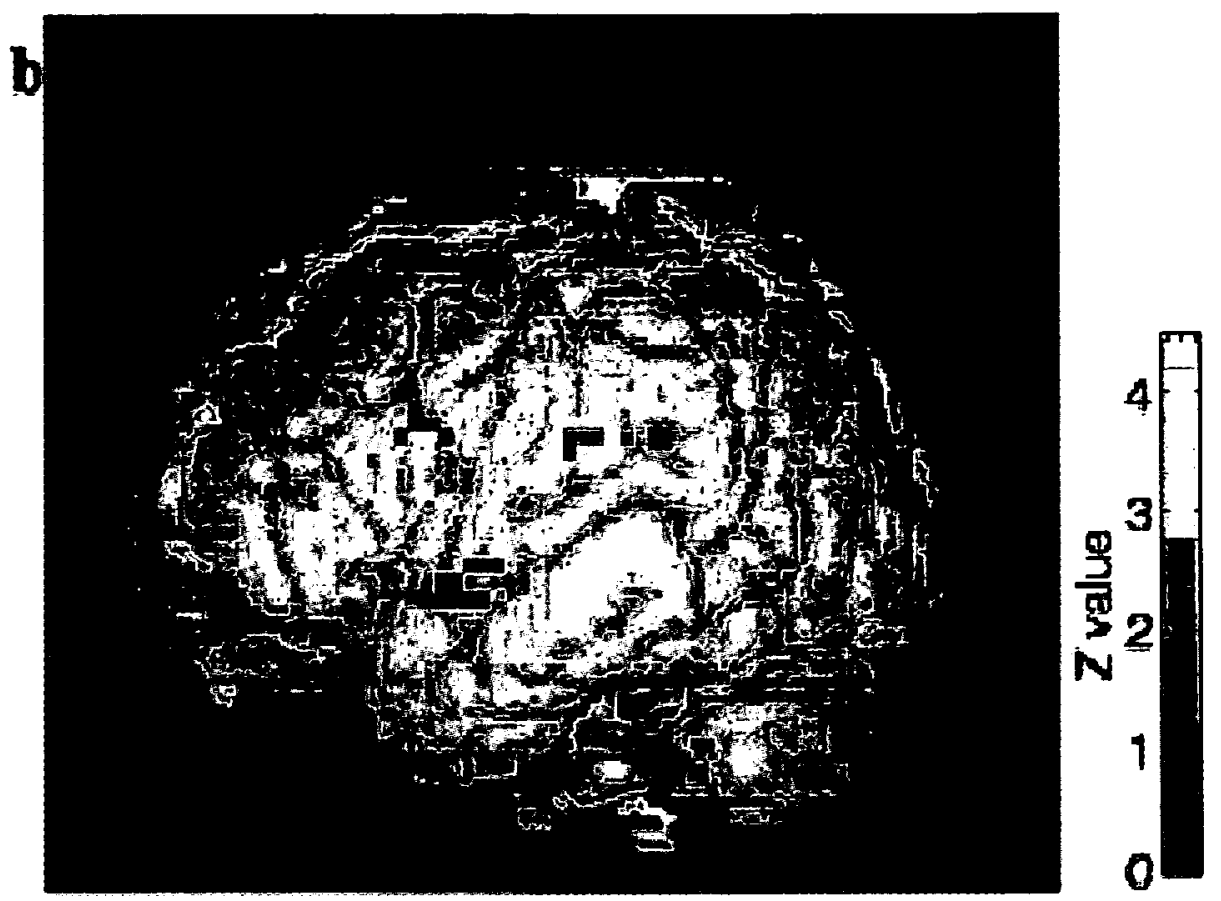

Figure 1 : Activation du gyrus temporal supérieur droit et gauche, du cortex pariétal inférieur gauche et du gyrus frontal moyen gauche pendant les périodes d'hallucinations auditives comparées aux périodes sans hallucinations, chez des patients atteints de schizophrénie. Les activations sont représentées sur des vues latérales droite (a) et gauche (b) du cerveau (d'après Lennox et coll. $(5))$.

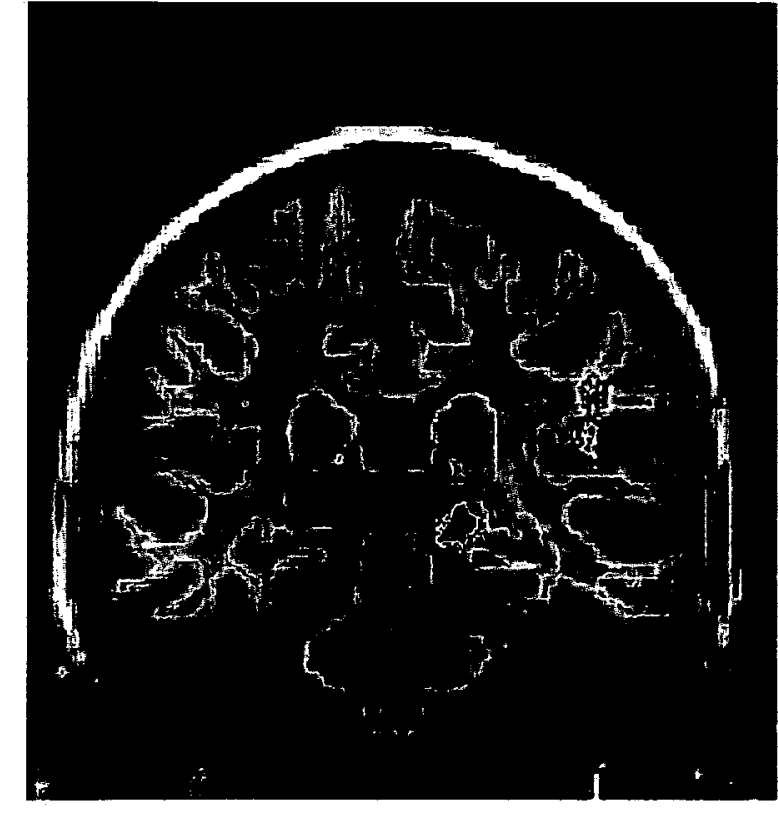

(2)

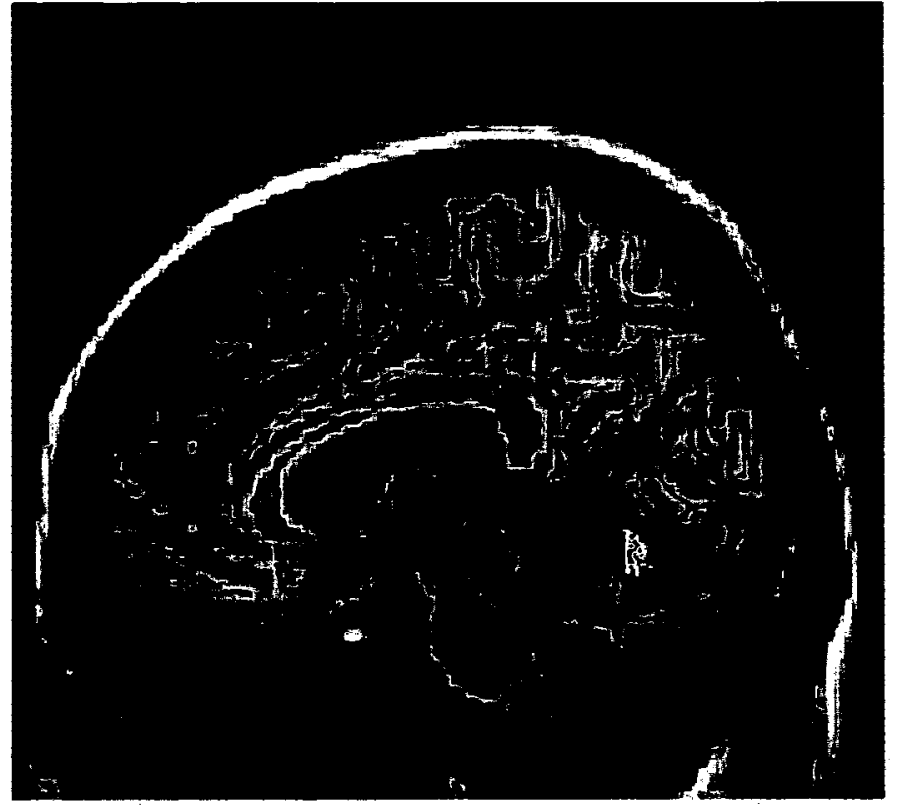

(b)

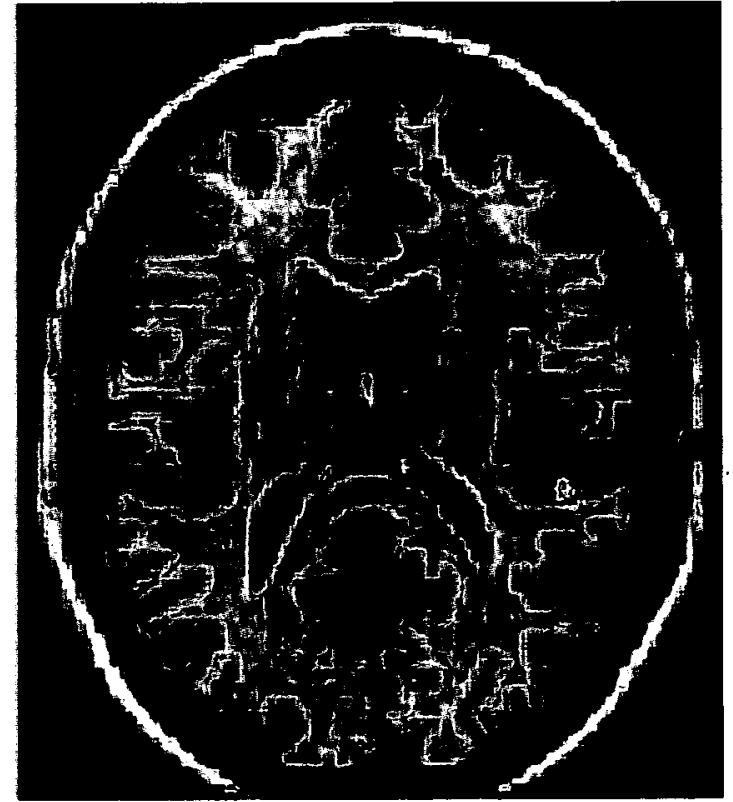

(c)

Figure 2 : Régions montrant les différences entre les patients schizophrènes et les sujets témoins lors de la production d'un haut débit de langage intérieur. Les régions corticales, en rouge, sont plus activées chez leș témoins que chez les patients schizophrènes (d'après Shergill et coll. (7)).

a) Coupe coronale montrant les différences d'activation dans le gyrus parahippocampique droit et le gyrus temporal supérieur/ lobe pariétal inférieur.

b) Coupe sagittale montrant l'activation du cortex cérébelleux latéral droit

c) Coupe axiale montrant l'activation du gyrus temporal supérieur droit. 
entre le cortex frontal et temporal serait responsable de l'impression fallacieuse que le langage intérieur produit par l'aire de Broca est en fait de provenance externe et serait perçu par le cortex auditif. Grâce à l'imagerie fonctionnelle on peut comprendre quelles aires cérébrales jouent un rôle dans la perception ou la production du langage, y compris de langage intérieur.

Pour étudier les anomalies du contrôle du langage intérieur, McGuire et coll. (6) ont comparé trois groupes de patients schizophrènes avec et sans hallucinations et des sujets témoins. Trois taches étaient proposées lire des mots silencieusement (tache contrôle); penser des phrases ayant la forme suivante : «tu es...», «tu es un...» et se terminant par des mots présentés, en utilisant sa voix intérieure habituelle (discours intérieur), penser des phrases se terminant par des mots présentés, en imaginant que ces phrases sont prononcées par quelqu'un d'autre (imagerie verbale auditive). Les patients hallucinés présentaient en particulier une diminution de l'activité cérébrale au niveau du gyrus temporal moyen gauche (AMS) (aire 21) et de l'aire motrice supplémentaire (aire 6) quand ils imaginaient quelqu'un d'autre parler, alors que les témoins et les autres patients présentaient une augmentation d'activité dans ces zones. Le temporal ne s'activerait pas parce que le sujet recevrait son discours comme quelque chose d'étranger. Les hallucinations correspondraient ainsi à un discours intérieur (activité de la zone de Broca) qui ne serait pas en lui-même anormal, mais ne pourrait pas être attribué au sujet du fait des anomalies des aires qui servent normalement à déterminer l'origine du discours (AMS et gyrus temporal moyen gauche). Shergill et coll. (7) proposent un atlas anatomo-fonctionnel des hallucinations acoustico-verbales chez le patient atteint de schizophrénie. La Figure 2 montre qu'il existe des différences entre les patients schizophrènes et les sujets tẹmoins lors de la production d'un haut débit de langage intérieur. Les régions corticales sont plus activées chez les témoins que chez les patients schizophrènes.

Shergill et coll. (7) ont observé un défaut d'activation des régions frontales avec une atténuation des modulations de ces aires sur les aires temporales et cérébelleuses, régions impliquées dans l'autocontrôle (ou contrôle de l'action).

L'hypothèse selon laquelle les hallucinations acoustico-verbales émanent d'une interprétation erronée de la parole intérieure et l'hypothèse impliquant une anomalie de l'activation du cortex auditif primaire, ne seraient par ailleurs pas exclusive l'une de l'autre (8).

\section{Conclusion}

Ces études explorent plusieurs voies qui pourront pro- bablement orienter les recherches thérapeutiques vers la modulation de l'activité de ces structures. L'imagerie fonctionnelle serait alors un outil d'évaluation des effets pharmacologiques mais aussi de certaines psychothérapies. Les informations anatomo-fonctionnelles apportées par l'imagerie pourraient permettre l'émergence et l'évaluation de nouvelles stratégies thérapeutiques à venir.

\section{Références}

1. Verdoux H., van Os J. Psychotic symptoms in non-clinical populations and the continuum of psychosis. Schizophr Res. 2002 ; 54(1-2) : 59-65.

2. Ey H. Traité des hallucinations. Paris : Masson, 1978.

3. Franck N., Thibaut F. Hallucinations. Encycl. Méd. Chir. (Editions Scientifiques et Médicales Elsevier SAS, Paris), Psychiatrie. 2003 ; 37-120-A-10,18 p.

4. Bar K.J., Gaser C., Nenadic I., Sauer H. Transient activation of the somatosensory area in painful hallucinations shown by fNMR. Neuroreport. $2002 ; 13$ (6) : 805-8.

5. Lennox B.R., Park S.B., Medley I., Morris P.G., Jones P.B. The functional anatomy of auditory hallucinations in schizophrenia. Psychiatry Res. $2000 ; 100(1)$ : 13-20.

6. McGuire P.K., Silbersweig D.A., Wright I., Murray R.M., David A.S., Frackowiak R.S., Frith C.D.. Abnormal monitoring of inner speech: a physiological basis for auditory hallucinations. Lancet. 1995 ; 346(8975) : 596-600.

7. Shergill S.S., Brammer M.J., Fukuda R., Williams S.C., Murray R.M., McGuire P.K. Engagement of brain areas implicated in processing inner speech in people with auditory hallucinations. Br. J. Psychiatry. 2003 ; 182 : 525-31.

8. Bentaleb L.A., Beauregard M., Liddle P., Stip E. Cerebral activity associated with auditory verbal hallucinations: a functional magnetic resonance imaging case study. J Psychiatry Neurosci. $2002 ; 27(2): 110-5$. 